\title{
La bioética como plataforma de los cuidados espirituales en la persona humana con discapacidad motora
}

\author{
Belkis Labina Zambrano Mapariz, ${ }^{\mathrm{a}}$ Yulays Carolina Canabal Roa, ${ }^{\mathrm{b}}$ Engelis Marian Yañez Capiello, ${ }^{\mathrm{a}}$ Eva Mendoza, ${ }^{\mathrm{b}}$ \\ Gregoriana Mendoza ${ }^{\mathrm{c}}$
}

Zambrano MBL, Canabal RYC, Yañez CEM, Mendoza E, Mendoza G. La bioética como plataforma de los cuidados espirituales en la persona humana con discapacidad motora. Cuid salud, ene-jun 2015; 2(1):148-155.

\section{RESUMEN}

Propósito: analizar la bioética como plataforma de los cuidados espirituales en la persona humana con discapacidad motora. Metodología: cualitativa, método fenomenológico, informantes: seis personas de las parroquias del Municipio Maracaibo, República Bolivariana de Venezuela. Técnicas: la observación, autorreportaje, entrevista semiestructurada, filmación-grabación y notas de campo, se obtuvieron 18 corpus discursivos. Resultados: surgieron las siguientes categorías: "Discapacidad" "Persona humana, trato discriminatorio, narra la forma como es tratada, la persona humana con discapacidad motora por la sociedad", "miedo", "familia", "ambiente", la accesibilidad relacionada con las herramientas necesarias que le brinda la sociedad a la persona humana con discapacidad motora "la autorrealización, del Ser o del yo social"; "Ser reconocido como persona, Volver a leer su vida", "Búsqueda de un sentido". A modo de Conclusión: los cuidados espirituales identificaron las necesidades humanas altas y las necesidades espirituales en los informantes, se abordaron las realidades antropológicas, la corporeidad humana, el ser espiritual, el crecimiento espiritual y el ser para la comunión, se desarrolló el juicio moral, la praxis de la bioética, la correcta conducta, el respeto a la vida, las virtudes, el deber, la felicidad y el buen vivir. La bioética fue la plataforma de los cuidados espirituales para la persona con discapacidad motora.
Zambrano MBL, Canabal RYC, Yañez CEM, Mendoza E, Mendoza G. Bioethics as platform of spiritual care to human being with motor disability. Cuid salud, ene-jun 2015; 2(1):148-155.

\begin{abstract}
Purpose: To analyze bioethics as platform of spiritual care to human being with motor disability. Methodology: Qualitative study, phenomenological method, key informants: six person from Maracaibo Municipality, Bolivarian Republic of Venezuela. Techniques: observation, self-report, semi-structured interviews, filmrecording and field notes, 18 were performed discursive corpus. Results: Emerged the following categories: "Handicap" "The Human Person, discriminatory treatment, the human being with motor disability discriminated by society "fear", "family ", "environment", accessibility related with the necessary tools that society provides the individual with motor disabilities "self-realization of Being or Social Being", "social"; "To be recognized as a person, Reread his life" Finding a sense. Conclusions: Spiritual cares identified spiritual needs in the informants, addressed anthropological realities, human corporeal, spiritual being, spiritual growth and being for communion, moral judgment, the practice of bioethics, right conduct, respect for life, virtue, duty, happiness and good living was developed. Bioethics was the platform of spiritual care for people with motor disabilities.
\end{abstract}

Keywords: Bioethics, nursing, care, spirituality.

Palabras clave: bioética, enfermería, atención, espiritualidad.

\footnotetext{
a Técnico Superior en Enfermería, estudiante Enfermería del décimo semestre de la Universidad del Zulia.

${ }^{\text {b }}$ Doctora en Ciencia Gerenciales, Especialista en Cuidados Críticos, Licenciada en Enfermería Docente Titular de la Facultad de Medicina de la Universidad del Zulia.

${ }^{\mathrm{c}}$ Doctora en Innovaciones Educativas. Especialista en Metodología de la Investigación, Licenciada en enfermería, Docente Titular de la Facultad de Medicina de la Universidad del Zulia
} 


\section{INTRODUCCIÓN}

La discapacidad es una realidad humana, está presente en gran parte de la población mundial y afecta a niños, jóvenes y adultos. A nivel planetario el número de personas con discapacidad es alto y aumenta día a día, resaltando que un $15 \%$ presenta discapacidad motora transitoria que limita sus posibilidades de desplazamiento. ${ }^{1}$

En el continente africano existen niños con discapacidad, quienes son discriminados debido al estigma social que predomina y la percepción negativa que se tiene de los niños y niñas con discapacidad, la cual se ve agravada por tres factores: su género, el tipo de discapacidad y el grado de dependencia, ${ }^{2}$ siendo además excluidos de los otros niños y solo una pequeña parte frecuenta la escuela y unos pocos culminan la educación.

En Europa, el continente más poblado después de Asia, África y América, la cifra de personas con discapacidad es mayor a 411 millones. ${ }^{3}$ En América, se estima que entre 140 y 180 millones de personas sufren de algún tipo de discapacidad, cifra que bordea los 85 millones de personas en América Latina. Destacando que la discapacidad y el envejecimiento se incrementan en América Latina y el Caribe; solo entre el 20 y $30 \%$ de niños con discapacidad asisten a la escuela en la Región. ${ }^{1}$

En México, el 5,13\% de la población tiene algún tipo de dificultad ${ }^{4}$ y los adultos mayores poseen al menos una limitación para realizar sus actividades de autocuidado. ${ }^{5}$ Según el Instituto Brasileño de Geografía y Estadística 12,7 millones de personas $(6,7 \%)$ de la población poseen un tipo de deficiencia severa. ${ }^{6}$ En la Región, Chile es el país con un envejecimiento moderadamente avanzado y el $62,9 \%$ padece de enfermedades

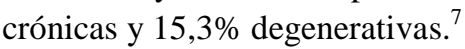

En la República Bolivariana de Venezuela, según el Instituto Nacional de Estadística del total de personas que en el Censo de Población 2011 respondieron a la pregunta de deficiente condición o discapacidad (27 019 815), el 5,38\% declaró tener al menos una discapacidad. De éstos el $0,9 \%$ tenía discapacidad motora en las extremidades superiores e inferiores. ${ }^{8}$

Ante esta realidad, en el 2008 se crea la Misión José Gregorio Hernández, institución dedicada al estudio de la población con algún tipo de discapacidad. ${ }^{8}$ Siendo que en el estado de Zulia existen 1724 personas con alguna discapacidad. Por estas cifras, Venezuela es considerado un país con un gran número de personas con discapacidad; destacando que en los indicadores la discapacidad intelectual presenta el más alto índice de demanda educativa ocupando el $88,6 \%$ respecto a la población total inscrita en el programa de atención a la persona con discapacidad, seguida de la discapacidad motora con un $8,9 \%$ y la auditiva con 2,5\%. Destacando que el estado de Zulia concentró 265546 declaraciones de personas con discapacidad, resultando la entidad Federal con más declaraciones en el país.

Frente a esta situación, surgen las siguientes interrogantes: ¿Cuáles son las medidas espirituales que acepta la persona humana con discapacidad motora desde el punto de vista ético? ¿Cómo la bioética establece una plataforma de los cuidados espirituales a una persona humana con discapacidad motora?

Para las autoras, la ética es fundamental para una disciplina que tiene como eje central al ser humano, ya que estudia la moral, la virtud, el deber, la felicidad y el buen vivir; lo que permite proporcionar cuidados con principios y visión espiritual arraigados en una correcta conducta humana de vida, considerando la situación de vulnerabilidad de la persona con discapacidad motora, quien requiere de medidas especiales $\mathrm{y}$ espirituales para desenvolverse en forma autónoma. Bajo esta perspectiva, se asume un enfoque transdisciplinario desde la bioética, la cual conjuga los dilemas éticos, especialmente cuando se refiere a la vida de las personas. Aunque es una disciplina teórica, en la práctica se sustenta en los derechos humanos y la dignidad de la persona, principalmente de aquellas diferentes, como las personas con discapacidad motora.

Desde la visión espiritual, se consideran tres aspectos primordiales: el significado y el propósito, la voluntad de vivir y, la fe en uno, en los demás, en Dios. Entendiendo a la espiritualidad como el significado de vida o el deseo intenso por vivirla; así siendo, los cuidados espirituales vivenciados con estas personas y sus familias constituyen un recurso profundo y potente de autonomía, en el que juega un papel importante la creencia religiosa. Los familiares serán capaces de brindar cuidado espiritual cuando apoyan a la persona con discapacidad a explorar lo que es significativo para ellas en sus vidas y según busquen formas de ajustarse a sus limitaciones. 
Es importante, especialmente en el estado de Zulia, promover cuidados espirituales en las personas con discapacidad para así mantener de forma efectiva la salud, por medio de la bioética como plataforma de los cuidados espirituales. El propósito de este estudio fue analizar la bioética como plataforma de los cuidados espirituales en la persona con discapacidad motora, considerando el aporte teórico, crítico reflexivo de la Teoría del Modelo Social de la Discapacidad, ${ }^{9}$ Teoría Bioética del Trato Humano ${ }^{10}$ y Teoría de los Cuidados Espirituales como Innovación Educativa. $^{11}$

\section{BASES TEÓRICO-REFERENCIALES}

El Modelo Social de la Discapacidad tiene como finalidad estudiar la exclusión de las personas con discapacidad en la sociedad. Un aspecto fundamental del modelo es la capacidad de tomar decisiones y la oportunidad de vivir la vida al máximo. ${ }^{9}$ Con relación a la Teoría Bioética del Trato Humano, ésta tiene como premisas: la autoconciencia, la conciencia epistemológica, la conciencia psicológica y la conciencia moral; las cuáles ayudan a interpretar la realidad existencial, la vida diaria, la relación con las demás personas y las respuestas sobre la discapacidad. En esta teoría, el liderazgo ético y la existencia de la dimensión interpersonal del hombre se destacan por la palabra activa, mediante la escucha del otro y la expresión del otro. ${ }^{10}$

Por último, la Teoría Fenomenológica de los Cuidados Espirituales es una innovación educativa que busca entender lo que se muestra y cómo se muestra en la conciencia de la persona la vida y la discapacidad motora. Presenta tres momentos: frente a las necesidades, cuando se recibe el diagnóstico y en la relación enfermera/ usuario. ${ }^{11}$ La finalidad de esta estrategia es desarrollar la conciencia pre-reflexiva y la conciencia emotiva a través de la educación, en la que el profesional de salud y los familiares perciban la discapacidad motora como un objeto trascendente; así, la explicación de la discapacidad se da no en el mundo sensible sino en el mundo real, expresado en la existencia y en la existencialidad del hombre. Otro aspecto de los cuidados espirituales es la existencia en lo existencial y en la existencialidad. ${ }^{11}$

\section{MARCO METODOLÓGICO}

\section{Abordaje epistemológico}

La investigación se enfoca en el paradigma cualitativo, al explorar las relaciones sociales y describir la realidad tal como la experimenta la persona humana con discapacidad motora. ${ }^{12}$

El método fue el fenomenológico, que hace énfasis en la experiencia, se fundamenta en la esencia de las cosas. ${ }^{12}$ El método se fundamenta en el referencial filosófico creado por Husserl, citado por Martínez, ${ }^{13}$ que se sustenta en "ir a las cosas mismas", lo que quiere decir, permitir ver lo que se muestra, tal como se muestra por sí mismo.

\section{Área de estudio y descripción de la información}

El estudio fue realizado durante el periodo enero-diciembre 2013. Previamente se solicitó permiso y consentimiento informado de los (3) consejos comunales pertenecientes al Municipio Maracaibo del estado: del Museo, los Dulces y el Modelo. Dos informantes pertenecían a la parroquia Luis Hurtado Higuera, en la localidad el Museo. Otros dos fueron de la parroquia San Isidro en el barrio los Dulces del Sector la Ponderosa y dos de la parroquia Venancio Pulgar en el barrio el Modelo del Sector el Marite.

\section{Elección de informantes clave}

Fue conformado por tres (3) informantes con discapacidad motora $y$ tres cuidadores $(n=6)$, quienes se seleccionaron mediante visitas domiciliarias previo permiso de los consejos comunales según área de influencia de las parroquias. En el perfil laboral, tres se desempeñaban como amas de casa, uno trabajaba en estética y los otros dos no trabajaban.

\section{Técnicas de recolección de la información}

Una vez obtenido los permisos, se realizó el diseño de la entrevista semiestructurada dirigida a la persona con discapacidad motora y otra dirigida al cuidador informal. Se elaboró el diseño del autorreportaje. Los informantes firmaron la hoja de consentimiento previa explicación del estudio y su objetivo.

Como técnicas fueron utilizadas la entrevista, observación no participante y visitas domiciliarias. La entrevista fue realizada primero en las parroquias de cada comunidad; luego se realizaron la transcripción de los corpus discursivos, identificándose nueve corpus los cuáles se verificaron con los propios informantes. En un segundo momento, se realizaron 15 visitas domiciliarias a los informantes, a fin de desarrollar los cuidados espirituales desde la Teoría Fenomenológica, $^{11}$ cuya duración fue de 15 
minutos. Pasado esta fase, nuevamente fueron realizados entrevistas con cada informarte clave.

Paralelamente fueron utilizadas otras técnicas como el autorreportaje, filmación y grabación, notas de campo; destacando que la filmación es particularmente válida para el descubrimiento y la validación. Asimismo, documenta la comunicación y el comportamiento no verbal, ayudando mucho en la interpretación de la información, que luego fue validada por otro investigador.

\section{Técnicas de registro y análisis de información}

En el estudio se transcribieron 18 corpus discursivos, nueve en el primer momento de las entrevistas y otros nueve en el desarrollo de los cuidados espirituales. En ellos se relatan las vivencias, experiencias, emociones, kinesis de las personas con discapacidad motora, las de sus familiares y las de los investigadores.

Para interpretar la información se usaron como métodos el hermenéutico-dialéctico y el fenomenológico. Una vez reproducida la información, se identificaron las categorías en los autorreportajes y entrevistas; la selección se realizó utilizando plumones de colores. Finalmente, se realizó la construcción de dos cuadros semánticos en dos momentos, antes y después de los cuidados espirituales. Construyéndose luego los mapas representacionales en cada momento.

\section{Consideraciones Éticas}

En el estudio se consideraron los principios bioéticos fundamentales como la autonomía, la no maleficencia, la justicia y la beneficencia mediante el consentimiento informado. Este proyecto fue presentado ante las jurisdicciones de los consejos comunales en el comité comunitario de personas con discapacidad, cuyas funciones son: gestionar la elaboración de proyectos, metas y actividades que atiendan las necesidades sectoriales de las personas con discapacidad, aprobar y llevar la gestión de los proyectos, planes y programas de las diferentes actividades relacionadas con los asuntos sociales de las personas con discapacidad.

\section{RESULTADOS Y DISCUSIÓN}

Una de las categorías de mayor frecuencia en el primer momento fue la "Discapacidad", como se describe: "el hecho que estemos así no nos implica que no podamos hacer cosas que los buenos que tienen sus dos piernas completas lo hacen".
Además, emergió la categoría "Persona humana", en la que manifestaban "yo me considero una persona que quiero y tengo tantas metas yo quiero seguir adelante quiero trabajar". "que la propia persona pueda desenvolverse por sí mismo".

Entre las categorías de mediana frecuencia se encuentran "trato discriminatorio", "miedo" y "familia". En el trato discriminatorio, la persona narra cómo es tratada, catalogada por la sociedad por su discapacidad motora "he experimentado que hay mucha discriminación en la sociedad", "la sociedad ve como algo extraño algo anormal que no debiera ni siquiera de existir".

En la categoría "miedo" emergió "tengo miedo, para ir a la universidad, no se salir a la calle en este momento", "me siento mal para poder, quisiera trabajar". Asimismo, en la categoría "familia" se identificaron "una carga familiar en cuanto a mi esposo y para mi hijo", "quiero ayudar a mi familia".

Así también, se describen las categorías de menor frecuencia como el "ambiente" relacionado con todo el entorno de la persona humana con discapacidad motora: "el entorno debe estar con bastante luz, suficiente iluminación, una buena naturaleza que pueda tener árboles cerca ya sea en el patio de su casa o en lugar donde habite”.

Emergió igualmente, la categoría "accesibilidad" referido a las herramientas necesarias que le brinda la sociedad para poder desplazarse en el ambiente social, como "en la mayoría de los centros comerciales hay rampas donde tiene la facilidad de subir y bajar ya que en otras partes solo hay escaleras". Asimismo, surgió la categoría "creer en un ser superior" que tiene mucho a ver con la parte espiritual de la persona humana con discapacidad: "pero somos iguales ante la presencia de Dios", "que Dios los bendiga”. Además de la categoría "amor", que expresa la trascendencia que siente la persona hacia el otro, en esta se identificó: "yo me complazco con atenderlo, para demostrarle a ellos que no son menos que nadie y que si pueden salir adelante y que son tan importantes”. En último lugar emergió la categoría "derecho" que está relacionada con los valores y principios éticos que deben tener todas las personas, en este se encuentra: "derecho a la vida al estar en la sociedad con derecho a participar de todos los derechos". 
En el segundo momento, después de los cuidados espirituales se destacaron "la autorrealización” como expresión de motivación y expresiones altruistas: "me siento autorrealizada cuando le sirvo a los demás cuando le soy útil a otras personas, cuando me arreglo para salir a hacer cualquier cosa". Asimismo, "Del Ser o del yo" que expresaba "yo me amo y me valoro no me da vergüenza nada, siempre y cuándo lo que haga no me perjudique ni este dañando a otra persona". Y "social", "me gusta tratar de comprender a los demás me gusta compartir con mis vecinos porque me siento complacida de expresarles lo importante que son".

Además emergieron categorías relacionadas con las necesidades espirituales "Ser reconocido como persona": "sí, me identifican como persona, me quieren tal cual como soy, me gusta hacer amistades". "Volver a leer su vida": "recuerdo cuando sali embarazada de mi primera hija cuando sentí sus primeras pataditas en mi vientre fue muy lindo". "Búsqueda de un sentido": "Cristo es quien le da sentido a mi vida", "el estar viva para servir a los demás". Además de "Librarse de la culpa" expresado en "yo me sentí culpable cuando mi hija menor se fue de la casa pero ya me libré de la culpa porque ahora está feliz cómoda junto a su esposo”. "Reconciliación": "con la persona que yo considero que me reconciliaría seria con mi esposo, para poder cerrar ese ciclo en mi vida”, "con mi hermana ya que por medio de la casa donde vivíamos quedamos peleadas”.

De todas las categorías emergidas y considerando el contexto del estado de Zulia como uno de los veintitrés estados de Venezuela de todo el territorio venezolano, siendo la quinta entidad de mayor superficie en Venezuela. Zulia cuenta con una población total de 3.821.068 habitantes, el $5,02 \%$ de los habitantes presenta alguna discapacidad, de los cuales un $0,6 \%$ pertenece a una población indígena. Según el censo de 2011 las personas con discapacidad motora, representa un $0,9 \%$; con discapacidad mental $0,5 \%$; con discapacidad auditiva $0,4 \%$; con discapacidad visual $1,7 \% .{ }^{14}$ De acuerdo a las cifras descritas, tanto en Venezuela como a nivel mundial existe gran número de personas con discapacidad, en las cuales la gran mayoría son niños, seguido de los ancianos.

En este orden de ideas, se observa que las categorías coinciden con lo expuesto por la Teoría del Modelo Social de la Discapacidad, ${ }^{9}$ en la que se analiza las experiencias de las personas con discapacidad, la identificación de las barreras sistémicas, las actitudes negativas y la exclusión de la sociedad; factor principal que contribuye a inhabilitar a las personas afectadas por la discapacidad. $\mathrm{Si}$ bien las variaciones físicas pueden causar limitación funcional física $o$ discapacidad, éstas no tienen que conducir a una incapacidad, a menos que la sociedad los ignore, independientemente de sus diferencias individuales. La lucha por la igualdad en los derechos, el empoderamiento, la capacidad de tomar decisiones y la oportunidad de vivir la vida al máximo es lo fundamental en esta teoría. En el estudio se evidencia que los informantes clave en los dos momentos, los cuidados espirituales expresan la necesidad de ser en la sociedad; llámese en la familia, amigos y compañeros de labor; identificándose una necesidad de empoderarse de su existencia en todos los ámbitos sociales.

Para las investigadoras es prioritario la toma de conciencia en la sociedad venezolana con relación a los derechos y la calidad de vida de las personas con discapacidad, además del empoderamiento de su vida y ser considerados como seres humanos con potencialidades espirituales, psicológicas y emocionales, donde exista una conjunción de la persona humana: ser ahí y ser con, permitiendo que se produzca la trascendencia en las actividades cotidianas, en las ecológicas y sociales. Además, las personas con discapacidad también deben tomar conciencia de lo que se les ofrece en los cuidados espirituales, de sus derechos y deberes como ciudadanos venezolanos, facilitarles la inserción social en su núcleo familiar y comunitario. Asimismo, la reincorporación a los estudios universitarios para lograr sus metas, objetivos en la vida, su participación en la toma de decisiones familiares, comunitarias y así mejorar su calidad de vida, profundizar en su ser y en la trascendencia e ir más allá de sí mismos. Sentirse persona humana.

Esto, también concuerda con lo expresado en la Convención Internacional sobre los Derechos de las Personas con Discapacidad y su Protocolo Facultativo, aprobados en el seno de la Asamblea General de las Naciones Unidas, cuya esencia es promover, proteger y asegurar los derechos inherentes a la dignidad de las personas. La igualdad en el reconocimiento como personas ante la Ley (artículo $\left.12^{\circ}\right)^{15}$ la igualdad de acceso a la justicia (artículo $13^{\circ}$ ) el respeto al hogar, la familia y la igualdad de trato en todas las cuestiones 
relacionadas con el matrimonio, la familia y la paternidad (artículo $23^{\circ}$ ).

En el país, la Ley de la persona humana con discapacidad publicada en la Gaceta Oficial $\mathrm{N}^{\mathrm{o}}$ $38.598,{ }^{16}$ especifica que el objetivo es regular los medios y mecanismos para garantizar el desarrollo integral de las personas con discapacidad de manera plena y autónoma, considerando sus capacidades, el disfrute de los derechos humanos y el logro de su integración a la vida familiar y comunitaria, promoviendo su participación directa como ciudadanos y ciudadanas plenos de derechos y, la participación solidaria ante la sociedad y la familia.

También a través de los comités de trabajo de los consejos comunales, del comité comunitario de personas con discapacidad en Venezuela y en el estado de Zulia, en la que este comité ejerce la vigilancia social dentro del Consejo Comunal para que se cumplan los derechos y deberes, estableciendo programas y proyectos que atiendan de manera integral y que garanticen a las personas con discapacidad, la inclusión y equiparación de oportunidades en relación a otros miembros de la comunidad, a su vez, promueve las diferentes actividades culturales, deportivas y recreativas para estas personas vinculándose, con los comités de protección e igualdad social, recreación y deporte.

La economía comunal, para establecer los planes, metas y actividades relacionados con los asuntos sociales que conlleven a mejorar la calidad de vida de las personas con discapacidad y sus familias, considera los art: $19^{\circ}, 20^{\circ}, 21^{\circ}, 22^{\circ}, 60^{\circ} \mathrm{y}$ $62^{\circ}$ de la Constitución de la República Bolivariana de Venezuela; la Ley para las Personas con Discapacidad, la Ley Orgánica de los Consejos Comunales y el Proyecto Nacional Simón Bolívar (II eje Suprema Felicidad Social). ${ }^{16}$

Igualmente, la Teoría Fenomenológica de los Cuidados Espirituales como Innovación Educativa, ${ }^{11}$ evidencia los enunciados epistémicos caracterizados por la sistematicidad sobre el ser, el sentido del ser y, la existencia; es decir, buscar lo que se muestra y cómo se muestra en la conciencia de la persona sobre la vida, el fenómeno de la discapacidad motora: "Me acepto como soy con mi discapacidad en realizar mis labores a pesar de [...]".

Los cuidados espirituales identifican las denominadas necesidades humanas altas y las necesidades espirituales de los informantes, partiendo desde las bases antropológicas, la corporeidad humana, el ser espiritual, el crecimiento espiritual y el ser para la comunión: "me considero espiritual me siento bien como soy porque $[. .$.$] ".$

Esta perspectiva también se ajusta con el Artículo $5^{\circ}$ de la Ley de la persona humana con discapacidad en la República Bolivariana de Venezuela, entendiendo que la discapacidad es la condición compleja del ser humano constituida por factores biopsicosociales que evidencian una disminución o supresión, temporal o permanente, de alguna de sus capacidades sensoriales, motrices o intelectuales, que puede manifestarse en ausencias, anomalías, defectos, pérdidas o dificultades para percibir, desplazarse sin apoyo, ver u oír, comunicarse con otros, o integrarse a las actividades de educación o trabajo en la familia y con la comunidad, que limitan el ejercicio de derechos, la participación social y el disfrute de una buena calidad de vida, o impiden la participación activa de las personas en las actividades de la vida familiar y social; sin que ello implique necesariamente incapacidad o inhabilidad para insertarse socialmente. ${ }^{16}$

Respecto a la Teoría de la Bioética del Trato Humano, ${ }^{10}$ hace referencia al juicio moral que expresan las actitudes afectivas, las preferencias, las decisiones de los informantes ante el fenómeno social de la discapacidad; el desarrollo del juicio moral o el tipo de conciencia de la persona ante el fenómeno, éste se incluye en el proceso de socialización con la familia, el colegio, la comunidad, el estado, la economía. Aspectos que son importantes a considerar en la proporción y adecuación de la calidad de vida en las personas humanas con discapacidad.

En la praxis, la bioética busca proveer los principios para la correcta conducta humana, respeto a la vida humana y no humana (animal y vegetal), además del ambiente en el que pueden darse condiciones aceptables para la vida. "a mí me parece que los valores es algo que se inculca [...], a mis hijos le he enseñado el respeto, el amor, la responsabilidad [...]".

La Ley sobre la discapacidad hace alusión al trato social y protección familiar: este artículo es pertinente ya que la persona humana con discapacidad es etiquetada, marcada de por vida por el solo hecho de tener capacidades diferentes. ${ }^{16}$ Esto ha generado, actitudes de rechazo, lástima, 
angustia e incomodidad, asimismo, conductas de sobreprotección familiar, lo que conduce a tratarlos como personas incapaces de valerse por sí mismas, potenciarse y desarrollarse.

Sin embargo, la mayor preocupación es la discriminación y falta de igualdad de oportunidades, en cuanto a educación, trabajo, salud, barreras arquitectónicas, sociales y culturales, que impiden su real integración a la comunidad. Se manifiesta una aproximación y afirmación que la bioética como plataforma de los cuidados espirituales para la persona humana con discapacidad motora promueve una actitud moral, vivencia de virtudes, cumplimiento del deber, el

\section{Correspondencia:}

Eva Mendoza

Correo electrónico: evadegraterol@yahoo.com

\section{REFERENCIAS BIBLIOGRÁFICAS}

1. Organización Mundial de la Salud. Banco Mundial. Resumen: Informe mundial sobre la discapacidad [internet]. Malta: Organización Mundial de la Salud; 2011 [citado 17 de jun 2013]. 27 laudas. Disponible en: http://www.who.int/disabilities/world_report/2 $\underline{011 / \text { accessible_es.pdf?ua }=1}$

2. Plan Internacional. Los niños con discapacidad en África Occidental se enfrentan a abusos generalizados [homepage de internet]. ONG Plan Internacional: España; sf [actualizado sf, citado 20 de ago 2013]. Disponible en: http://plan-espana.org/prensa-y-

publicaciones/noticias-ong/ninos-discapacidaddiscriminacion-africa-plan/

3. INE: Instituto Nacional de Estadística. Encuesta de discapacidad, autonomía personal y situaciones de dependencia 2008 [internet]. España: INEbase; 2008 [actualizado 2012; citado 10 de may 2013]. Disponible en: http://www.ine.es/jaxi/menu.do?type=pcaxis\& path $=\% 2 \mathrm{Ft} 15 \% 2 \mathrm{Fp} 418 \&$ file=inebase

4. INEGI: Instituto Nacional de Estadística y Geografía. Población total y su distribución porcentual según condición y causa de limitación en la actividad para cada tamaño y sexo [internet]. México: INEGI; 2010 [actualizado 26 de jun 2013; citado 10 de jul 2013]. Disponible en: cuéntame.inegi.org.mx/población/discapacidad .aspx desarrollo de la felicidad y el buen vivir en la persona humana con discapacidad motora.

A modo de conclusión se puede decir que los cuidados espirituales identificaron las necesidades humanas altas y las necesidades espirituales en los informantes, abordándose las realidades antropológicas, la corporeidad humana, el ser espiritual, el crecimiento espiritual y el ser para la comunión, se desarrolló el juicio moral, la praxis de la bioética, la correcta conducta, el respeto a la vida, las virtudes, el deber, la felicidad y el buen vivir. La bioética fue la plataforma de los cuidados espirituales para la persona con discapacidad motora.

5. ENSANUT: Encuesta Nacional de Salud y Nutrición. Resultados nacionales 2012 [internet]. México: ESANUT; oct 2012 [citado 20 may de 2013]. 36 p. Disponible en: http://ensanut.insp.mx/doctos/ENSANUT2012 Sint_Ejec-24oct.pdf

6. IBGE: Instituto Brasileño de Geografía e Estadística. Censo Demográfico 2010[internet]. Brasil: IBGE; nov 2011 [citado 10 de oct 2013]. 17 p. Disponible en: http://ibge.gov.br/home/estatistica/populacao/c enso2010/default.shtm

7. CELADE: Centro Latinoamericano y Caribeño de Demografía. Discapacidad [internet]. Chile: CELADE; 2009 [citado 10 de may 2013]. 7 p. Disponible en: http://www.eclac.cl/celade/noticias/paginas/3/3 7763/Susana_Schkolnik.pd.

8. Instituto Nacional de Estadística. Gerencia General de Estadísticas Demográficas. Población con discapacidad en Venezuela, según el Censo 2011. Boletín Demográfico [serie en internet]. Dic 2013 [citado 20 de may 2013]; 1-8. Disponible en:http://www.ine.gov.ve/documentos/SEN/me nuSEN/pdf/subcomitedemografica/Documento s2014/Boletin_de_Mesa_Tecnica_de_Discapac idad_2014.pub_ver_4_nov.pdf

9. López GM. Modelos teóricos e investigación en el ámbito de la discapacidad. Hacia la 
incorporación de la experiencia personal [internet]. Córdova: López GM; 2005 [citado 18 de jul 2013]. 17 p. Disponible en: www.invisibles.org.ar/.../modelos-teoricos-einv-en-el-ambito-discapacid.

10. Mendoza de GE. Teoría bioética del trato humano del liderazgo ético y la responsabilidad social de las organizaciones hospitalarias públicas [tesis doctoral]. Maracaibo Venezuela: Universidad Nacional Experimental Politécnica de las Fuerzas Armadas de la República Bolivariana de Venezuela (UNEFA), 2011.

11. Mendoza G. Cuidados espirituales como innovación educativa. España: EAE Editorial Academia Española; 2012. 57 p.

12. Martínez M. El paradigma emergente. Hacia una nueva teoría de la racionalidad científica. México: Trillas; 2005. 263 p.

13. Martínez M. La nueva ciencia: su desafío, lógica y método. México: Trillas; 2002. 271 p

14. República Bolivariana de Venezuela. Instituto Nacional de Estadística. Gerencia General de Estadística demográfica. boletín demográfico la población con discapacidad en Venezuela, según el censo 2011 disponible en: http://www.ine.gov.ve/documentos/SEN/menu SEN/pdf/subcomitedemografica/Discapacidad/ PoblacionDiscapacidadVzla.pd

15. Naciones Unidas. Convención sobre los Derechos de las Personas con Discapacidad y Protocolo Facultativo [internet]. Ginebra: Naciones Unidas; sf. [citado 17 de jun 2013]. 42 p. Disponible en: http://www.un.org/disabilities/documents/conv ention/convoptprot-s.pdf

16. La Asamblea Nacional de la República Bolivariana de Venezuela. Ley para la persona con discapacidad [internet]. Venezuela: Asamblea Nacional de la república Bolivariana de Venezuela; may 2014 [citado 17 de jun 2014]. 27 p. Disponible en: http://www.minvih.gob.ve/faami/phocadownlo adpap/Leyes-faami/discapacidad.pdf 